\title{
Dietary recommendations for patients with rheumatoid arthritis: a review
}

\author{
This article was published in the following Dove Press journal: \\ Nutrition and Dietary Supplements \\ 16 June 2012 \\ Number of times this article has been viewed
}

\author{
Luis Vitetta' \\ Samantha Coulson' \\ Janet Schloss' \\ Shoshannah L Beck' \\ Robert Allen ${ }^{2}$ \\ Avni Sali ${ }^{2}$ \\ 'Centre for Integrative Clinical and \\ Molecular Medicine, The University \\ of Queensland School of Medicine, \\ Brisbane, ${ }^{2}$ National Institute of \\ Integrative Medicine, Melbourne, \\ Australia
}

\begin{abstract}
Dietary interventions can assist with the management of disease symptoms that accompany rheumatoid arthritis (RA), such as pain, tender swollen joints, stiffness, and associated disability and disease progression. Dietary interventions have gained widespread appeal for both clinicians and RA patients. Interventions that promote self-help through education can have significant benefits for patients as they negotiate pain and musculoskeletal disability. There is substantial scientific evidence that demonstrates patients diagnosed with RA may benefit from dietary interventions; however, recent systematic reviews remain uncertain about the therapeutic efficacy of dietary manipulation for RA due to clinical trials with a high risk of bias. However, dietary interventions with plausible therapeutic activity may be indicated for reducing RAassociated symptoms, including elimination of foods that may trigger an allergic or intolerant response, introduction of known anti-inflammatory dietary compounds and correction of food, or drug-induced gastrointestinal tract microbiota abnormalities and permeability.
\end{abstract}

Keywords: diet, rheumatoid arthritis, vegetarian, vegan, Mediterranean, fish oils, probiotics

\section{Introduction}

Rheumatoid arthritis (RA) is a chronic autoimmune, inflammatory disease with unclear pathophysiology processes. RA may be multiple diseases, currently defined by some common clinical manifestations, and there may not be a single predominant mechanism of initiation or perpetuation. ${ }^{1}$ The current view is that inflammation and tissue destruction in the rheumatoid synovium results from complex cell-cell interactions, initiated by antigen-presenting cells and CD $4^{+} \mathrm{T}$ cells. ${ }^{1}$ This is followed by macrophage activation and the release of proinflammatory cytokines such as interleukin-1 and tumor necrosis factor- $\alpha$ (TNF $\alpha$ ) that stimulate synovial fibroblasts and chondrocytes in articular cartilage to secrete enzymes that degrade proteoglycans and collagen, leading to tissue destruction. ${ }^{1}$ Autoimmunity and the overall systemic and articular inflammatory load drive the destructive progression of the disease. ${ }^{2} \mathrm{RA}$ is characterized by joint pain, tenderness, stiffness and swelling, rheumatoid nodules, and destruction of synovial joints, leading to severe disability, reduced quality of life, and premature mortality. ${ }^{2}$ Serology is positive for such autoantibodies as rheumatoid factor and anticitrullinated protein antibody, which can precede the clinical manifestation of RA by many years. ${ }^{2}$

Furthermore, rheumatic conditions including RA are associated with an increased prevalence of gastrointestinal tract (GIT) symptoms, particularly dyspepsia (epigastric pain and burning, postprandial fullness, bloating, early satiety, nausea, and belching ${ }^{3}$ ), mucosal ulceration, and altered bowel habits (constipation/diarrhoea), 
which are associated with reduced quality of life. ${ }^{4,5}$ With much interest, evidence has been reported that patients with RA have significant modification of the intestinal microbiota that differs from that of healthy control patients. ${ }^{6-8}$ RA patients demonstrate significantly less Bifidobacterium species and bacteria of the Bacteroides-PorphyromonasPrevotella group, and a decrease in lactobacteria with various reports of high and low Clostridium species. The abundance of opportunistic enterobacteria and staphylococci were also noted to be elevated. Research is now exploring the hypothesis that intestinal microbiota may participate in the aetiopathogenesis of RA. ${ }^{7,8}$

Current research is highlighting the need to define subsets of RA by genetic or serologic markers to enable more concise treatment options for patients. Understanding the multiple predisposing or protective genetic factors provides hope for a new direction, ${ }^{1}$ and genetic interaction with GIT microbiota may be the key in developing new treatments or preventive measures based on modulation of the GIT microbiota. The host genotype may guide both the composition of GIT microbiota and immune responses against microbes, and in individuals susceptible for RA, the arthritogenic bacterial antigens may pass from intestines to the joints, causing prolonged immunological response and articular inflammation. ${ }^{7}$

For decades, dietary manipulation has been used by patients diagnosed with RA, in the hope that it may improve their symptoms. ${ }^{9}$ Dietary manipulation is still widely used today. There are various commonly used dietary programs utilized for treating RA, such as medically supervised fasting (7-10 days) followed by a vegetarian or vegan diet, vegetarian and Mediterranean diets, elemental diet plans, and elimination diets that are thought to possibly prevent the autoimmune response associated with the pathophysiological process of the disease. A vegetarian eating plan may be varied, as it can be either strictly a vegan diet or a lacto-ovo-vegetarian diet, which allows consumption of dairy products and eggs. The mechanisms of action that dietary manipulation may provide to produce health benefits may result in the rescuing of a potentially dysbiotic GIT that is distorted by sustained proinflammatory metabolites. Plant-based and elimination diets may reregulate the inflammatory process by inducing a more balanced GIT microbiota, leading to a downregulation of inflammation locally and systematically. ${ }^{10}$ The effector mechanism may involve a consequent change in the profile of the GIT microbioata that subsequently elaborate secondary metabolites from such diets, which may be seen as beneficial for the host. Vegetarian, vegan, and Mediterranean-type diets are high in such dietary compounds as those found in vegetables and legumes (eg, phytochemicals, unsaturated fats) which can maintain a regulated anti-inflammatory effect by interacting positively with the GI microbiota, counteracting dysbiosis. ${ }^{11}$

\section{Methodology}

We searched the Cochrane Central Register of Controlled Trials, Medline, EMbase, AMED, Cinahl, and reference lists of relevant articles up to December 2011. The selection criteria included randomized controlled trials or single- or double-blinded controlled clinical trials where the effectiveness of dietary manipulation was evaluated (Table 1). Dietary supplement studies that included fish oils and probiotics were also included, as the overall aim of this review was to explore the role that diets and functional foods may have in adjusting GIT function and reregulating local inflammatory processes that then could positively influence RA. Studies with individual micronutrients (eg, zinc vitamins) were not included.

\section{Dietary interventions Fasting/vegetarian/vegan diet}

Clinical experience suggests that fasting followed by a vegetarian diet may help patients with RA. ${ }^{11}$ A 2001 systematic review ${ }^{12}$ assessed the available scientific evidence, as patients frequently sought dietary advice, and exclusive pharmacological treatment for RA was often declined due to the side effects the patients may experience. The results of the controlled studies, which reported follow-up data for at least 3 months after fasting, were quantitatively pooled. Thirty-one reports of fasting studies in patients with RA were found. Only four controlled studies investigated the effects of fasting and subsequent diets for at least 3 months. The pooling of these studies showed a statistically and clinically significant beneficial long-term effect. The available evidence tends to suggest that fasting followed by a vegetarian diet may be significantly useful in the treatment of RA pain..$^{9,11,12}$

Once food is reintroduced after fasting, however, most patients with RA present with disease-activity relapses. The effect of a 7-10 day subtotal fast (partial nutrient intake during the fast consisted of herbal teas, garlic, vegetable broth, decoction of potatoes and parsley, and juice extracts from carrots, beets, and celery. No fruit juices were allowed. The daily energy intake during the fast varied between 800 and $1260 \mathrm{~kJ}$ ) followed by 1 year of a vegetarian diet compared to an ordinary diet was assessed in 53 patients. ${ }^{13}$ After 4 weeks of dietary changes, there was a significant improvement in the 


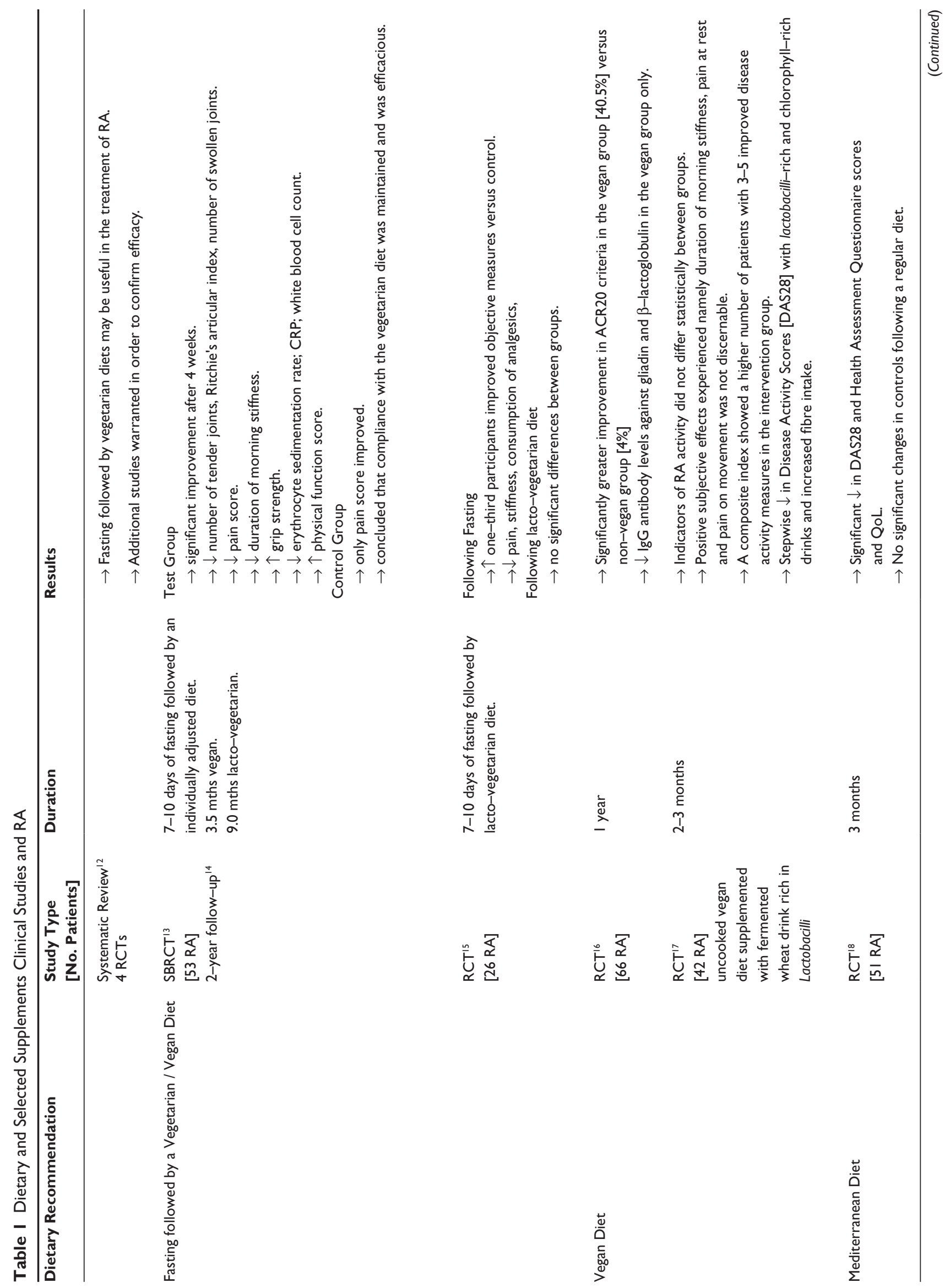




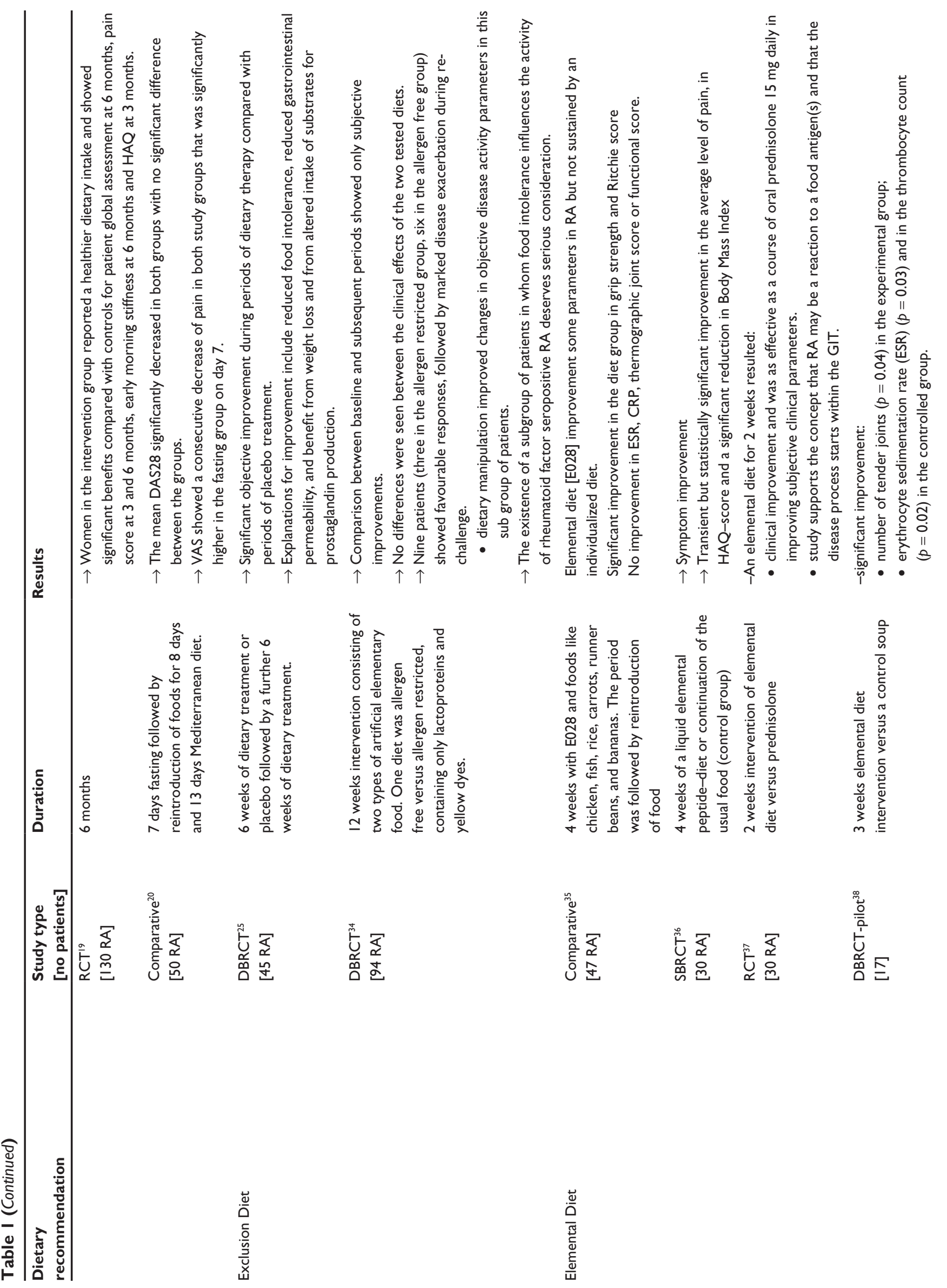




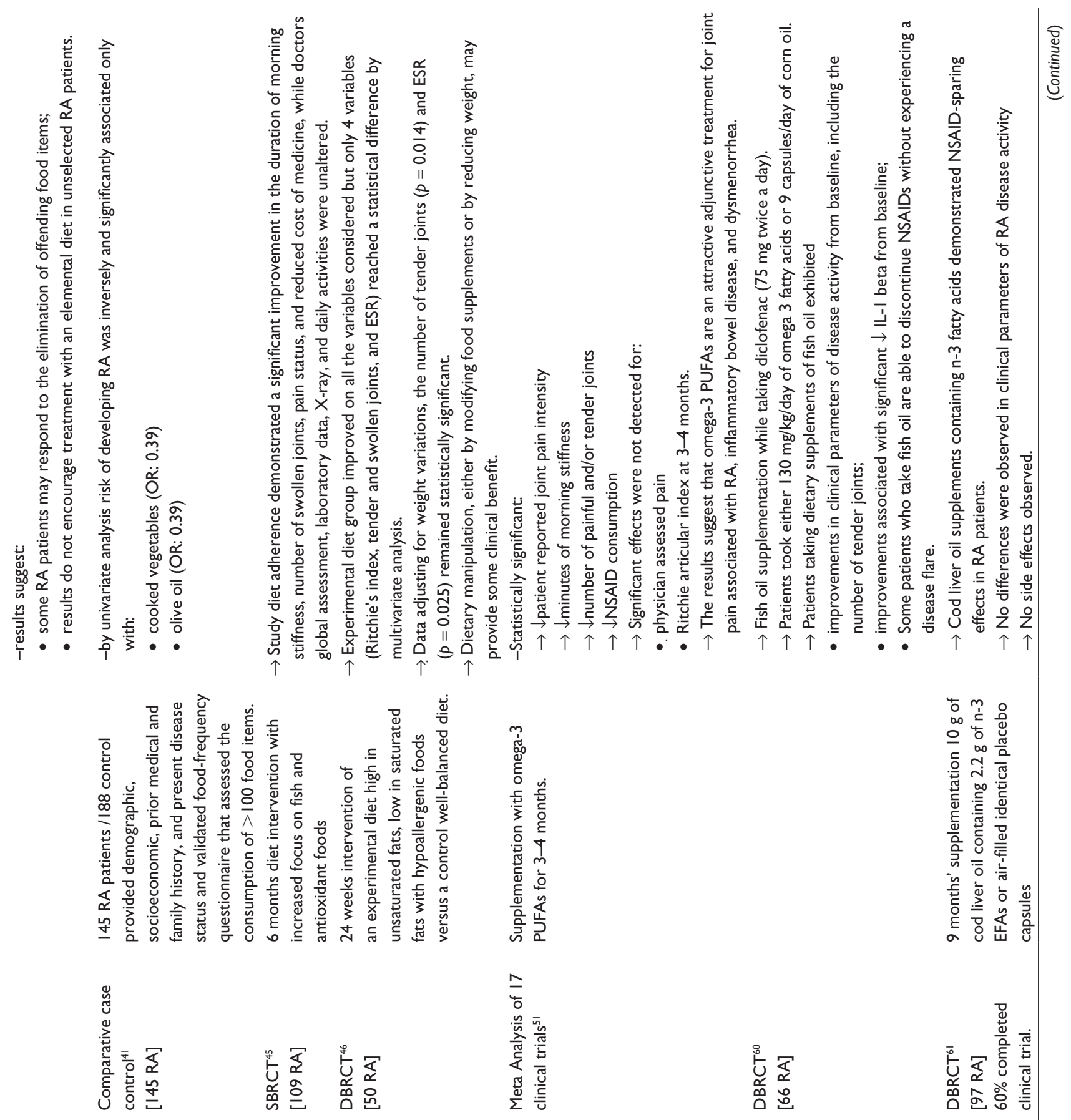

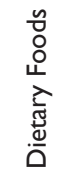

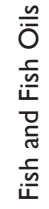




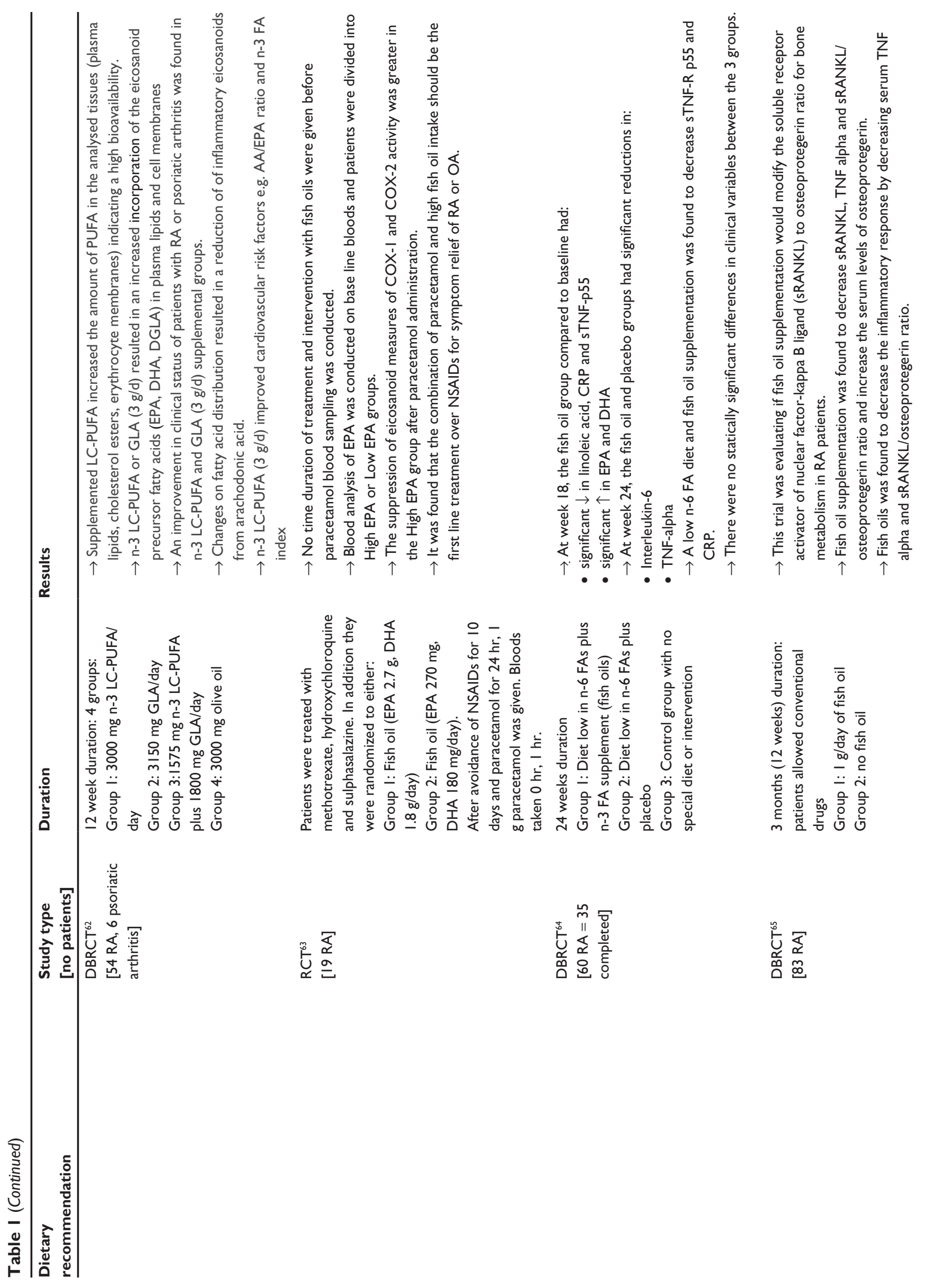



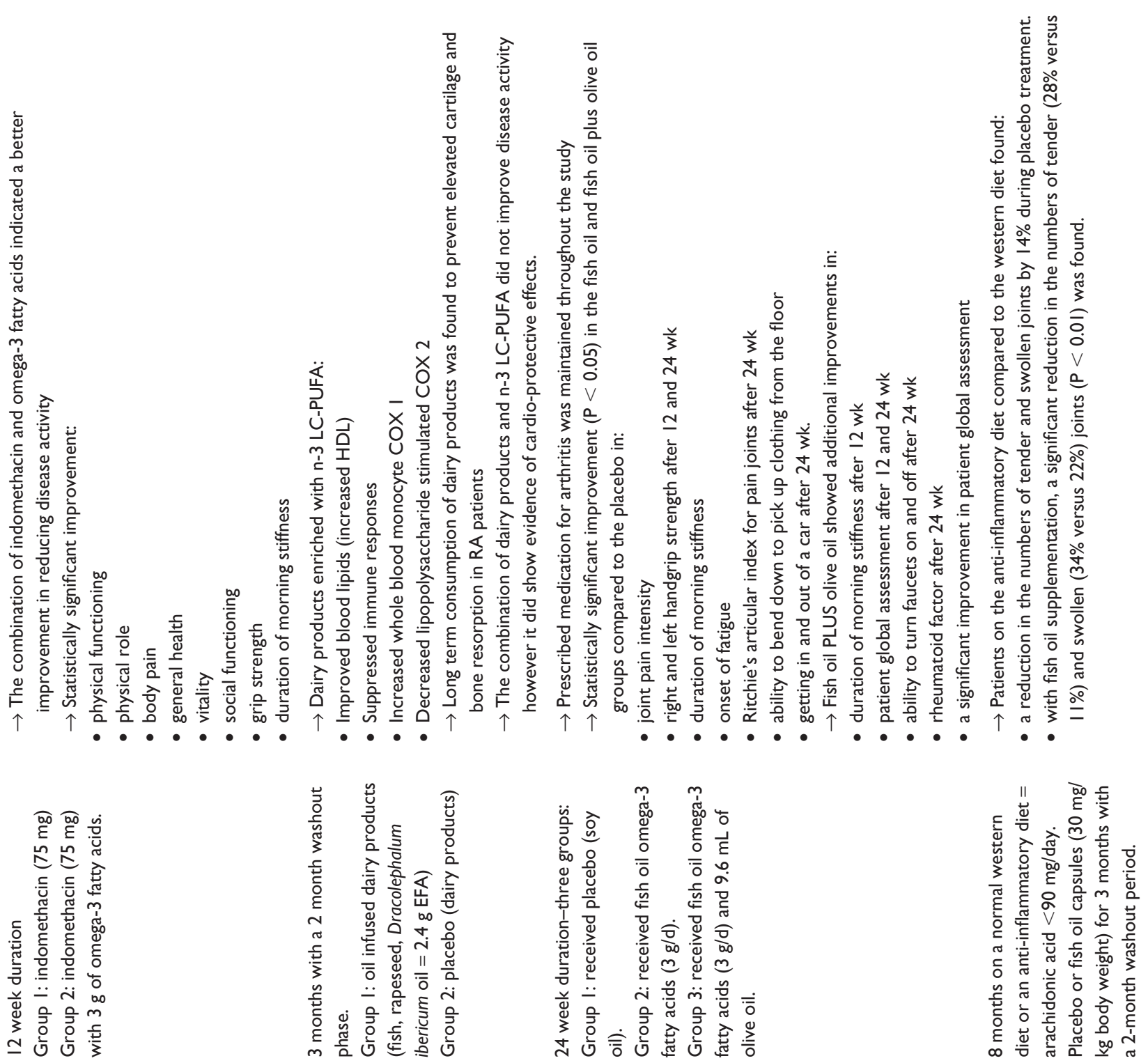

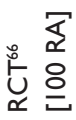

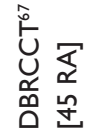

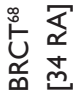

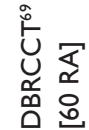


number of tender joints, Ritchie's articular index, number of swollen joints, pain score, duration of morning stiffness, grip strength, erythrocyte sedimentation rate (ESR), C-reactive protein, white blood cell count, and a health assessment questionnaire score. In the control group, only the pain score improved significantly. The benefits in the diet group were still present after 1 year, and evaluation of the whole course showed significant advantages for the diet group in all measured indices. It was further reported that improvements observed through the dietary manipulation could be sustained after 2 years. ${ }^{14}$ This dietary regimen may be a useful adjunct to conventional medical treatment for reducing pain in RA.

Research on fasting and vegan diets for RA remains variable, however, with an earlier study assessing a fasting/ vegan eating plan $(n=26)$ showing no significant differences at the end of the diet plan. ${ }^{15}$ Another clinical study investigating a vegan dietary intervention $(n=66)$ reported that dietary modification may be of clinical benefit for certain RA patients. The authors stated that this benefit may be related to a reduction in immunoreactivity to food antigens in the GIT that can be eliminated by the change in dietary consumption. ${ }^{16}$ A subsequent study $(\mathrm{n}=42)$ that tested the effects of an uncooked vegan diet rich in lactobacilli in RA patients reported that the uncooked vegan diet decreased subjective symptoms of RA compared to the control group. Moreover, it was reported that large doses of live lactobacilli consumed daily may also have positive effects on objective measures of RA. ${ }^{17}$ Nevertheless, additional randomized long-term studies are needed to confirm efficacy by improved methodologically convincing data. ${ }^{12}$

\section{The Mediterranean diet}

The Mediterranean diet reflects a dietary pattern that is largely characteristic of an anti-inflammatory diet. ${ }^{18-22}$ Typically, the diet comprises abundant plant foods (including fruits, vegetables, wholegrain cereals, beans, nuts, and seeds); minimally processed, seasonally fresh and locally grown foods; fish and poultry; and olive oil as the main source of lipid, with dairy products, red meat, and wine in low to moderate amounts. Thus, the diet is rich in long-chain $n-3$ polyunsaturated fatty acids (PUFAs) and oleic acid ( $n-9$ monounsaturated), phytochemicals, and unrefined carbohydrates. The Mediterranean diet has been linked with a significant reduction in all-cause morbidity and mortality, ${ }^{23}$ and therefore there is a propensity towards a plausible clinical improvement in RA inflammatory symptoms (that may promote the disablement process). A small number of studies have demonstrated efficacy (Table 1); however, a systematic review reported 
that the effects of dietary manipulation, including vegetarian, Mediterranean, elemental, and elimination diets, on RA still remain uncertain. ${ }^{24}$

\section{Exclusion diet}

An early double-blinded, placebo-controlled study that employed an exclusion diet in 53 RA patients demonstrated clinical improvement in joint pain and stiffness, ESR, and fibrinogen levels. ${ }^{25}$ All patients underwent a washout period from all previous therapy and then followed an exclusion phase for 1 week, in which only foods that were nonallergenic (foods that were unlikely to be ill tolerated) were allowed. Foods were then reintroduced one at a time to assess which foods caused symptoms. Foods that provoked an allergic reaction were then excluded from the diet. Darlington et $\mathrm{al}^{25}$ found that cereal foods induced the most reactions, with corn and wheat producing symptoms in over $50 \%$ of the patients. Cereal foods comprised four of the top seven symptom-inducing foods, with other foods such as pork, dairy, eggs, certain fruits, peanuts, lamb, coffee, and soy also causing intolerance and an exacerbation of RA symptoms in patients. ${ }^{26,27}$ The study concluded that it was possible to hypothesize that improvement included reduced food intolerance, reduced GIT permeability, and that the RA patients benefited from the observed weight loss and from a reduced intake of substrates for prostaglandin production. There is some evidence supporting dietary elimination therapy for RA, ${ }^{28-31}$ although inconsistencies have been noted and reported. ${ }^{32,33}$ Notwithstanding the contentious reporting, a small study that directly eliminated allergens from the diet provides a further insight that food intolerance may significantly influence the clinical activity of RA. This study enrolled 94 patients diagnosed with RA. ${ }^{34}$ The study postulated that there may be a subgroup of patients with RA in whom food intolerance influences the activity of rheumatoid factor seropositive RA and that there is a need for further serious consideration. This study further illustrates the notion that the GIT may have a significant role in influencing the progression of RA.

\section{Elemental diet}

Two clinical studies that investigated an elemental diet in 47 and 30 patients diagnosed with RA demonstrated partial efficacy (Table 1). ${ }^{35,36}$ The improvements were transient, however. Elemental diet (E028) is a hypoallergenic, proteinfree artificial diet consisting of essential amino acids, glucose, trace elements, and vitamins. The elemental diet is taken as an oral drink or administered via a nasogastric tube; however, due to poor tolerance it should only be considered as a temporary therapy. ${ }^{36}$ A small pilot trial $(n=30)$ investigated an elemental diet to oral prednisolone in a comparative study and reported that an elemental diet, when complied with for over 2 weeks, provided a clinical improvement in patients with active RA. ${ }^{37}$ This pilot study supported the hypothesis that RA may be a reaction to food antigens and that the disease process starts within the GIT. A further small pilot trial ${ }^{38}(\mathrm{n}=17)$ demonstrated that some RA patients may respond to the elimination of offending food items. However, the results do not encourage treatment with an elemental diet in unselected RA patients.

Research investigating the role that food antigens may have in promoting RA presents a complex inflammatory picture. Studies have reported that the production of cross-reactive antibodies is significantly increased in the GIT of many RA patients. ${ }^{39}$ Ingested food-related problems might reflect an adverse additive effect of multiple modest hypersensitivity reactions that are mediated by immune complexes that then may promote autoimmune reactions in the joints via a GIT joint axis. A recent review provides an insight into RA as a complex, polygenic, autoimmune disorder where genes have a role, but that environmental factors are required for disease manifestation. ${ }^{40}$ Furthermore, it suggested that disease pathogenesis may require a significant interplay with the GIT microbiome.

\section{Dietary foods}

Investigations of dietary elements considered to reduce the risk of RA have indicated that foods high in olive oil, omega-3-rich fish, fruit, vegetables, and beta-cryptoxanthins (found in red fruit and vegetables) have been reported to have a protective role for RA. ${ }^{21,41}$ A review of clinical trials on red meat, coffee, and alcohol consumption demonstrated contentious results, and no firm conclusions could be made as to their influence on RA. ${ }^{21}$ However, a further study of diet and risk indicated that although consumption of high-fat fish ( $\geq 8 \mathrm{~g}$ of fat/100 g fish) appeared to provide a reduced risk, medium-fat fish (3-7 g/100 g fish) was associated with an increased risk of RA. ${ }^{41}$ A prospective study suggested that cauliflower, broccoli, and other cruciferous vegetables and fruit were protective of RA. ${ }^{42}$ However, a further study indicated that fruit, coffee, olive oil, and meat intake showed no association with RA risk reduction, nor did intake of the vitamins $\mathrm{A}, \mathrm{E}, \mathrm{C}, \mathrm{D}$, and the minerals zinc, selenium, or iron. ${ }^{43}$ Additionally, a review of studies into the relationship between obesity and RA suggests that obesity may lead to less changes on radiography and better survival rates, although this needs to be confirmed. ${ }^{44}$

A dietary intervention study with 109 patients diagnosed with RA demonstrated significant improvement in 
the duration of morning stiffness, number of swollen joints, pain status, and reduced cost of medicine, in spite of doctors' global assessment, laboratory data, X-ray, and daily activities remaining unaltered. ${ }^{45} \mathrm{~A}$ further study with 50 patients ${ }^{46}$ diagnosed with RA reported that an experimental diet that was high in unsaturated fats, low in saturated fats with hypoallergenic foods versus a controlled well-balanced diet improved four RA-associated variables (Ritchie's index, tender and swollen joints, and ESR) reaching statistical difference by multivariate analysis. When the data were adjusted for weight variations, the number of tender joints $(P=0.014)$ and $\operatorname{ESR}(P=0.025)$ remained statistically significant. Hence, the study concluded that dietary manipulation, either by modifying food supplements or by reducing weight, may provide some clinical benefit.

\section{Omega-3 polyunsaturated fatty acids from fish and supplements}

Early descriptive observations demonstrated that populations such as the Greenland Eskimos, a group consuming a high-fat diet rich in omega-3 PUFAs containing high levels of eicosapentaenoic acid (EPA) and docosahexaenoic acid (DHA), were afforded protection from cardiovascular disease. ${ }^{47,48}$ Similarly, the Japanese population, reported to consume a diet relatively high in fish, presented lower rates of acute myocardial infarction and atherosclerosis. ${ }^{49,50}$ Hence, a clinical picture is presented that shows that EPA and DHA can significantly and favorably influence downregulation of proinflammatory profiles by reregulating the inflammatory response.

Omega-6 (n-6) and omega-3 (n-3) PUFAs are precursors of potent lipid mediators, termed eicosanoids, which play an important role in the regulation of inflammation. Eicosanoids derived from $n-6$ PUFAs (eg, arachidonic acid) have proinflammatory and immune-active functions, whereas eicosanoids derived from $n-3$ PUFAs (eg, EPA and DHA) have anti-inflammatory properties, traditionally attributed to their ability to inhibit the formation of $n-6$ PUFA-derived eicosanoids. While the typical Western diet has a high ratio of $n$-6 PUFAs compared with $n-3$ PUFAs, research has shown that by increasing the ratio of $n-3$ to $n-6$ fatty acids in the diet, and consequently favoring the production of EPA, or by increasing the dietary intake of EPA and DHA through the consumption of fatty fish or fish-oil supplements, reductions may be achieved in the incidence of many chronic diseases such as cardiovascular disease, inflammatory bowel disease, cancer, and RA that involves inflammatory processes. ${ }^{47}$

A meta-analysis covering 17 randomized controlled clinical trials (Table 1 ) demonstrated that $n-3$ fatty acid supplementation may be an effective adjunctive treatment for RA. ${ }^{51}$ The study showed a significant beneficial effect on RA pain, morning stiffness, number of painful and/or tender joints, and nonsteroidal anti-inflammatory drug (NSAID) consumption.

Several studies have reported that $n-3$ PUFAs have been demonstrated to exhibit immunomodulatory effects by changing the profiles of the eicosanoids produced and decreasing the levels of proinflammatory cytokines, via both lipid-mediator-related and non-lipid-mediator-related mechanisms, ${ }^{52,53}$ The content of marine $n$ - 3 fatty acids varies greatly according to the species of fish, the total fat content of the fish, and their geographical origin. ${ }^{54}$

Deepwater fish that have been described as oily (eg, tuna, salmon, mackerel, herring, and sardines) from cold climates have the highest content of EPA and DHA, since lipids are stored in the fish's flesh, whereas lean fish that store lipids in the liver (eg, cod) contain less EPA and DHA. One portion of cod provides approximately $0.3 \mathrm{~g}$ of EPA and DHA, compared to one portion of salmon, which provides approximately $1.5 \mathrm{~g}$ of EPA and DHA, and one portion of mackerel, which provides approximately 3 g. ${ }^{55}$ The EPA and DHA obtained from the flesh of oily fish or from the livers of lean fish is rich in $n-3 \mathrm{~s}$, and one fish-oil capsule from these sources consists of approximately $30 \%$ of these fatty acids. Hence, supplementation with a typical 1-g fish-oil capsule provides approximately $300 \mathrm{mg}$ of EPA and DHA, which is equivalent to the consumption of one portion of cod. However, it should also be noted that it has been reported that the intake of $n$ - 3 fatty acids in the absence of oily fish or from a fish-oil supplement is likely to be $<100 \mathrm{mg} /$ day. ${ }^{56,57}$ Consuming a daily supplement of $n-3$ fatty acids (standard fish-oil capsule per day) can increase $n-3$ levels fivefold (or more) in the absence of any other fish intake. ${ }^{58}$

Increased consumption of fatty fish or fish-oil supplements containing $n-3$ PUFAs increases the amount of these fatty acids and their metabolites in human immune cells and consequently changes the production of important mediators and regulators of inflammation and immune responses towards an anti-inflammatory profile. Since excessive intake of $n-6$ PUFAs, which is characteristic of Western diets, could potentiate inflammatory processes and consequently predispose to, or exacerbate, inflammatory diseases, increasing intake of fatty acids that elicit anti-inflammatory effects, such as $n$-3 PUFAs, could decrease the risk of many chronic diseases like arthritis and improve health. Based on the published health effects of $n-3$ PUFAs, recommendations 
have been made to increase dietary intake of these fatty acids, achieved by increasing consumption of oily fish or by consuming fish-oil supplements. ${ }^{59}$

An early clinical trial with patients $(n=66)$ taking dietary supplements of fish oil reported that the patients exhibited improvements in clinical parameters of disease activity from baseline. ${ }^{60}$ These included the number of tender joints, and these improvements were associated with significant decreases in levels of interleukin-1 beta from baseline. Some patients who took fish oils were also able to discontinue NSAIDs without experiencing a disease flare. A recent clinical trial that investigated cod liver oil supplementation versus placebo in RA (2.2 g EPA per $10 \mathrm{~g}$ cod liver oil) reported that cod liver oil supplements containing $n-3$ fatty acids demonstrated NSAID-sparing effects in RA patients. ${ }^{61}$ Numerous other clinical studies reported in a meta-analysis ${ }^{51}$ and listed in Table 1 further support the role that fish fats may have on improving disease outcome in patients diagnosed with RA. ${ }^{62-69}$

\section{Gastrointestinal influences in RA}

Two of this group of researchers have previously reviewed the nutritional supplement and herbal medicine scientific/ medical literature for osteoartritis and $\mathrm{RA}^{70}$ and it will not be repeated here. Suffice to add that importantly, we have focused on the GIT and briefly on probiotics and prebiotics in this review. The commensal (normal microflora, indigenous microbiota) GIT microbiome and functional foods such as probiotics and prebiotics may have a useful role as pharmacobiotics in RA.

\section{Gastrointestinal complaints in RA patients}

Gastrointestinal symptoms are reportedly common among patients with rheumatic disorders, and medications alone are not responsible for the high prevalence, suggesting that the underlying chronic rheumatic condition predisposes the patient to GIT symptoms. ${ }^{71-73}$ Prescribed medications used for the treatment of rheumatic disorders, including NSAIDs, steroids, and disease-modifying drugs, have been associated with numerous adverse GIT events. ${ }^{72,73}$ In particular, dyspepsia, abnormal bowel habits (hard/loose stool), and abdominal bloating have been reported by RA and osteoarthritis patients. Interestingly, such symptoms are also reported by patients with irritable bowel syndrome, in which they have been associated with an altered profile of intestinal microbiota and unbalanced fecal organic acid levels. ${ }^{71,72}$

\section{Enteric microbiota in RA patients}

Different dietary profiles such as higher fat intake and lower fiber intake have recently been shown to be correlated with particular bacterial groups. ${ }^{74}$ Enterotypes appeared to be determined by long-term diet. Namely, the Bacteroides enterotype was positively associated with animal protein and saturated-fat intake, whereas the Prevotella enterotype was associated with predominantly plant-based nutrition with high carbohydrates and low meat and dairy consumption. Such studies may have important implications when considering dietary manipulation for RA. Furthermore, the association between the intestinal microbiome and disease activity may have implications for how diets can affect RA.

A recent study has investigated the fecal microbiota in early RA. ${ }^{6}$ This study showed that RA patients had significantly less bifidobacteria and bacteria of the BacteroidesPorphyromonas-Prevotella group, Bacteroides fragilis subgroup, and Eubacterium rectale-Clostridium coccoides group, indicating that intestinal microbes participate in the etiopathogenesis of RA. The aim of the work was to study colonic microbial biocenosis and colonizing ability of opportunistic bacteria in 32 patients with RA and 30 healthy subjects. RA was associated with significant modification of the intestinal flora. A decrease in lactobacteria and significant increases of enterococci, clostridia, and colibacteria were reported in RA patients showing reduced enzymatic activity, and an increase in opportunistic species. Also, symbiotic relationships between microorganisms were altered. The fraction of bifidobacteria, bacteroids, and lacto-positive colibacteria were reduced, while the abundance of opportunistic enterobacteria and staphylococci were increased. Opportunistic Enterobacteriaceae species were present in urine and nasal mucosa, suggesting their translocation from the intestines. Consistent with these observations, it has been further reported that changes in intestinal microflora and colonization by opportunistic bacteria enhance the risk of development of comorbid conditions in patients with RA. ${ }^{7}$

\section{Dietary-induced changes in fecal microflora in RA patients}

Clinical studies that have investigated a vegetarian $\operatorname{diet}^{75}$ or a form of uncooked vegan diet rich in lactobacilli ${ }^{76}$ have shown that these diets can change the fecal microbiota profile in RA patients. Changes in the fecal flora were associated with improvement in RA activity. Hence, could there be a role for probiotics as a pharmacobiotic ${ }^{77}$

The past six decades have seen a significant increase in the prevalence of autoimmune diseases. ${ }^{78}$ Hence, an autoimmune 
disease such as RA has forced a refocusing of research on the role the GIT and the microbiota may have on inducing autoimmune diseases. This was the catalyst that led to the formulation of the hygiene hypothesis. This hypothesis provides a biologically plausible explanation for the trend that implicates diminished exposure in early childhood to those normal infections that boost immune defenses. This deficit subsequently enhances the risk, for later life, of GIT inflammatory problems that disrupt normal/regulated GIT inflammatory responses and increases the susceptibility to developing autoimmune diseases. ${ }^{78}$

\section{Probiotic supplementation in RA patients}

A recent randomized double-blind clinical study has examined the effects of probiotic Lactobacillus rhamnosus GR-1 and $L$. reuteri $\mathrm{RC}-14$ capsules administered orally to $\mathrm{RA}$ patients. ${ }^{79}$ The study demonstrated that there was functional improvement seen within the probiotic group compared to placebo. In a further small clinical study, ${ }^{80}$ participants who received Bacillus coagulans GBI-30, 6086 experienced borderline statistically significant improvement in the Patient Pain Assessment score and statistically significant improvement in Pain Scale versus placebo. Moreover, compared with placebo, B. coagulans GBI-30, 6086 treatment resulted in greater improvement in patient global assessment and self-assessed disability; reduction in C-reactive protein; as well as the ability to walk 2 miles, reach, and participate in daily activities. In an additional double-blinded study, it was reported that Lactobacillus LGG was not better than placebo in the activity of RA. ${ }^{81}$

\section{Prebiotics}

Prebiotics are nondigestible food ingredients that are of benefit to the host by selectively promoting the growth and activity of a limited number of bacteria in the GIT. While probiotics provide bacteria to the GIT, prebiotics provide a food source for the growth of bacteria already in the gut. The predominant use of prebiotics is to potentiate the beneficial actions of bacteria in the GIT. ${ }^{10}$

\section{Synbiotics}

Synbiotics are functional foods that contain both a probiotic and prebiotic component. ${ }^{10}$ The rationale for such combination products is that together the formulation enhances the survival of probiotic bacteria in transit through the proximal GIT and improves commensal bacteria interactions with the probiotic in the large bowel, such as a stimulating effect on the growth of the endogenous flora. ${ }^{82}$ This effect may rescue the
GIT from a continued dysregulated inflammatory response that may increase the risk of developing an autoimmune disease such as RA.

\section{Discussion}

Musculoskeletal diseases can be incapacitating and detrimental to quality of life. Although effective pharmacologic treatments are available, the continuing high burden of disease and lost productivity affirms the need for further innovation. Diet, nutrition, and weight loss have shown promise in alleviating some of the disease burden of RA. ${ }^{83}$

Although the evidence presented from clinical trials for diets and foods is not robust, dietary advice does have a place in rheumatology as part of an integrative approach for the patient diagnosed with RA. The clinical trials presented in this review do demonstrate that a subset of patients will benefit from following a vegetarian, vegan, or Mediterraneanstyle diet, or by eliminating certain foods from their diet. There are observed similarities between the Mediterranean and the Paleolithic diets. Furthermore, a recent review has argued that Paleolithic diets are increasingly acknowledged as templates for healthy diets, partly because very low ageadjusted rates of cardiovascular disease and other nutritionrelated disorders have been observed among contemporary hunter-gatherers. ${ }^{85}$ Moreover, it has been reported that there are no obvious risks with avoiding dairy products, margarine, oils, refined sugar, and cereal grains, which provide $70 \%$ or more of the dietary intake in northern European populations. ${ }^{85}$ The importance of diet for human health may, amongst other things, be due to the influence of different nutrients on the health and integrity of the intestinal microbial flora. ${ }^{14}$ The intestinal microbial flora are crucial for the provision of important cues that assist the GIT innate immune system achieve full maturation. ${ }^{77}$ Dietary interventions have been assumed to have modulating effects on GIT permeability and integrity with clinical relevance to RA. ${ }^{15}$ Hence, it is plausible that dietary interventions that can unfavorably uncouple the GIT microbiome and innate immune system interchange may enhance the lifetime risk of developing RA.

The ever-increasing importance of the GIT microbiome in maintaining a regulated inflammatory response locally and systemically has grown further since the advent of the hygiene hypothesis. ${ }^{86} \mathrm{~A}$ recent review has outlined the historical clues that suggest a possible role for the microbiota in the pathogenesis of RA. ${ }^{40}$ A plausible hypothesis is that intestinal dysbiosis could lead, in a genetically predisposed individual, to a disruption in immune tolerance, followed by systemic immune disequilibrium that ultimately favors 
proinflammatory responses locally within the GIT. This then further escalates systemic reactions, resulting in peripheral tissue damage of the joints. Hence, the role of commensal bacteria and importantly of probiotic species in RA is a paradigm shift toward their implementation as pharmacobiotics. ${ }^{77}$

In order to ensure the induction of tolerance and protective immunity, discriminative responses are required to the commensal bacteria that make up the GIT microbiome in respective comparisons with pathogens. ${ }^{86}$ Namely, segmented filamentous bacteria can promote the differentiation of T-helper 17 cells, while certain Bifidobacterium, Lactobacillus, and Clostridium species can promote $\mathrm{T}_{\text {reg }}$ development. ${ }^{87,88}$ Hence, as postulated by the hygiene hypothesis, it may therefore seem most advantageous to maintain the GIT microbiome through dietary interventions that would promote GIT microbiome tolerance to environmental antigens.

There are no studies that have investigated the efficacy of either a prebiotic or synbiotic functional food in RA. However, a recent review does provide an insight into the biological plausibility of these functional foods assisting with the management of RA. Inflammation is a stereotypical physiological response to infections and tissue injury, as reported by Calder and colleagues. ${ }^{81}$

Furthermore, and importantly, an integrative approach that includes prudent nutritional practices (based on a Mediterranean-style diet that closely resembles a Paleolithic diet) with multistrain probiotics that synergistically downregulate proinflammatory responses may be a useful longterm management strategy for patients diagnosed with RA. ${ }^{84}$ Functional foods such as probiotics and prebiotics promoting gut-barrier function and anti-inflammatory responses may provide useful adjuncts as dietary therapies.

\section{Disclosure}

Professor Luis Vitetta has received industry funding for research into probiotics. All other authors report no conflicts of interest in this work.

\section{References}

1. Arend W. The pathophysiology and treatment of rheumatoid arthritis. Arthritis Rheum. 1997;40(4):595-597.

2. Aletaha D, Neogi T, Silman AJ, et al. 2010 Rheumatoid arthritis classification criteria: an American College of Rheumatology/European League Against Rheumatism collaborative initiative. Arthritis Rheum. 2010;62(9):2569-2581.

3. Corsetti M, Caenepeel P, Fischler B, Janssens J, Tack J. Impact of coexisting irritable bowel syndrome on symptoms and pathophysiological mechanisms in functional dyspepsia. Am J Gastroenterol. 2004;99(6):1152-1159.
4. Wolfe F, Kong S, Watson D. Gastrointestinal symptoms and health related quality of life in patients with arthritis. J Rheumatol. 2000;27(6): 1373-1378.

5. Chong V, Wang C. Higher prevalence of gastrointestinal symptoms among patients with rheumatic disorders. Singapore Med J. 2008;49(5):419-424.

6. Gul'neva M, Noskov SM. Colonic microbial biocenosis in rheumatoid arthritis. Klin Med (Mosk). 2011;89(4):45-48. Russian.

7. Vaahtovuo J, Munukka E, Korkeamäki M, Luukkainen R, Toivanen P. Fecal microbiota in early rheumatoid arthritis. J Rheumatol. 2008;35: 1500-1505.

8. Toivanen P. Normal intestinal microbiota in the aetiopathogenesis of rheumatoid arthritis. Ann Rheum Dis. 2003;62(9):807-811.

9. Smedslund G, Byfuglien MG, Olsen SU, Hagen KB. Effectiveness and safety of dietary interventions for rheumatoid arthritis: a systematic review of randomized controlled trials. J Am Diet Assoc. 2010;110(5): 727-735.

10. Vitetta L, Sali A. Probiotics, prebiotics and gastrointestinal health. Med Today. 2008;9:65-70.

11. Rayman MP, Pattison DJ. Dietary manipulation in musculoskeletal conditions. Best Pract Res Clin Rheumatol. 2008;22(3):535-561.

12. Muller H, de Toledo FW, Resch KL. Fasting followed by vegetarian diet in patients with rheumatoid arthritis: a systematic review. Scand J Rheumatol. 2001;30(1):1-10.

13. Kjeldsen-Kragh J, Haugen M, Borchgrevink CF, et al. Controlled trial of fasting and one-year vegetarian diet in rheumatoid arthritis. Lancet. 1991;338(8772):899-902.

14. Kjeldsen-Kragh J, Haugen M, Borchgrevink CF, Førre O. Vegetarian diet for patients with rheumatoid arthritis - status: two years after introduction of the diet. Clin Rheumatol. 1994;13(3):475-482.

15. Skoldstam L, Larsson L, Lindstrom F. Effects of fasting and lactovegetarian diet on rheumatoid arthritis. Scand $J$ Rheumatol. 1979;8:249-255.

16. Hafstrom I, Ringertz B, Spångberg A, et al. A vegan diet free of gluten improves the signs and symptoms of rheumatoid arthritis: the effects on arthritis correlate with a reduction in antibodies to food antigens. Rheumatology (Oxford). 2001;40(10):1175-1179.

17. Nenonen MT, Helve TA, Rauma AL, et al. Uncooked, lactobacilli-rich, vegan food and rheumatoid arthritis. Br J Rheumatol. 1998;37(3): 274-281.

18. Skoldstam L, Hagfors L, Johansson G. An experimental study of a Mediterranean diet intervention for patients with rheumatoid arthritis. Ann Rheum Dis. 2003;62:208-214.

19. McKellar G, Morrison E, McEntegart A. A pilot study of a Mediterranean-type diet intervention in female patients with rheumatoid arthritis living in areas of social deprivation in Glasgow. Ann Rheum Dis. 2007;66:1239-1243.

20. Abendroth A, Michalsen A, Lüdtke R, et al. Changes of intestinal microflora in patients with rheumatoid arthritis during fasting or a Mediterranean diet. Forsch Komplementmed. 2010;17(6): 307-313.

21. Pattison DJ, Harrison RA, Symmons DP. The role of diet in susceptibility to rheumatoid arthritis: a systematic review. J Rheumatol. 2004;31(7):1310-1319.

22. Pattison DJ, Symmons DP, Yound A. Does diet have a role in the aetiology of rheumatoid arthritis? Proc Nutr Soc. 2004;63(1):137-143.

23. Féart C, Pérès K, Samieri C, et al. Adherence to a Mediterranean diet and onset of disability in older persons. Eur J Epidemiol. 2011;26(9): $747-756$.

24. Hagen KB, Byfuglien MG, Falzon L, et al. Dietary interventions for rheumatoid arthritis. Cochrane Database Syst Rev. 2009;1: CD006400.

25. Darlington LG, Ramsey NW, Mansfield JR. Placebo-controlled, blind study of dietary manipulation therapy in rheumatoid arthritis. Lancet. 1986;327(8475):236-238.

26. Darlington LG, Ramsey NW. Review of dietary therapy for rheumatoid arthritis. Br J Rheumatol. 1993;32:507-514. 
27. Darlington L, Ramsey N. Dietary manipulation therapy in rheumatoid arthritis. In: Machtey I, editor. Progress in Rheumatology. Petah Tikva: Rheumatology Service, Golda Medical Centre; 1987;3:128-132.

28. Skoldstam L, Larsson L, Lindstrom F. Effects of fasting and lactovegetarian diet on rheumatoid arthritis. Scand J Rheumatol. 1979;8: 249-255.

29. Paganelli R, Levinsky RJ, Brostoff J, Wraith DG. Immune complexes containing food proteins in normal and atopic subjects after oral challenge and effect of sodium cromoglycate on antigen absorption. Lancet. 1979;1(8129):1270-1272.

30. Hicklin J, McEwen L, Morgan J. The effect of diet in rheumatoid arthritis. Clin Allergy. 1980;10:463.

31. Parke A, Hughes G. Rheumatoid arthritis and food. BMJ. 1981;282: 2027-2029.

32. Denman A, Mitchell B, Ansell B. Joint complaints and food allergic disorders. Ann Allergy. 1983;51:260-263.

33. Panush R, Carter R, Katz P. Diet therapy for rheumatoid arthritis. Arthritis Rheum. 1983;26:462-471.

34. van de Laar MA, van der Korst JK. Food intolerance in rheumatoid arthritis. I. A double blind, controlled trial of the clinical effects of elimination of milk allergens and azo dyes. Ann Rheum Dis. 1992;51(3):298-302.

35. Kavanagh R, Workman E, Nash P, et al. The effects of elemental diet and subsequent food reintroduction on rheumatoid-arthritis. Br J Rheumatol. 1995;34:270-273.

36. Holst-Jensen SE, Pfeiffer-Jensen M, Monsrud M, et al. Treatment of rheumatoid arthritis with a peptide diet: a randomized, controlled trial. Scand J Rheumatol. 1998;27:329-336.

37. Podas T, Nightingale JM, Oldham R, et al. Is rheumatoid arthritis a disease that starts in the intestine? A pilot study comparing an elemental diet with oral prednisolone. Postgrad Med J. 2007;83(976): $128-131$.

38. Haugen MA, Kjeldsen-Kragh J, Førre OA. Pilot study of the effect of an elemental diet in the management of rheumatoid arthritis. Clin Exp Rheumatol. 1994;12(3):275-279.

39. Hvatum M, Kanerud L, Hällgren R, Brandtzaeg P. The gut-joint axis: cross reactive food antibodies in rheumatoid arthritis. Gut. 2006;55(9):1240-1247.

40. Scher JU, Abramson SB. The microbiome and rheumatoid arthritis. Nat Rev Rheumatol. 2011;7(10):569-578.

41. Linos A, Kaklamani VG, Kaklamani E, et al. Dietary factors in relation to rheumatoid arthritis: a role for olive oil and cooked vegetables? AJCN 1999;70(6):1077-82.

42. Cerhan JR, Saag KG, Merlino LA, Mikuls TR, Criswell LA. Antioxidant micronutrients and risk of rheumatoid arthritis in cohort of older women. Am J Epidemiol. 2003;157:345-354.

43. Pedersen M, Stripp C, Klarlund M. Diet and the risk of rheumatoid arthritis in a prospective cohort. $J$ Rheumatol. 2005;32(7):1249-1252.

44. Magliano M. Obesity and arthritis. Menopause Int. 2008;14(4): $149-154$.

45. Hansen GV, Nielsen L, Kluger E, et al. Nutritional status of Danish rheumatoid arthritis patients and effects of a diet adjusted in energy intake, fish-meal, and antioxidants. Scand J Rheumatol. 1996;25(5): 325-330.

46. Sarzi-Puttini P, Comi D, Boccassini L, et al. Diet therapy for rheumatoid arthritis. A controlled double-blind study of two different dietary regimens. Scand J Rheumatol. 2000;29(5):302-307.

47. Wall R, Ross P, Fitzgerald G, et al. Fatty acids from fish: the antiinflammatory potential of long-chain omega-3 fatty acids. Nutr Rev. 2010;68(5):280-289.

48. Bang HO, Dyerberg J. Lipid metabolism and ischemic heart disease in Greenland Eskimos. Acta Med Scand. 1976;200:69-73.

49. Menotti A, Kromhout D, Blackburn H, et al. Food intake patterns and 25-year mortality from coronary heart disease: cross-cultural correlations in the Seven Countries Study. The Seven Countries Study Research Group. Eur J Epidemiol. 1999;15:507-515.
50. Holub BJ. Clinical nutrition: 4. Omega-3 fatty acids in cardiovascular care. CMAJ. 2002;166:608-615.

51. Goldberg RJ, Katz J. A meta-analysis of the analgesic effects of omega-3 polyunsaturated fatty acid supplementation for inflammatory joint pain. Pain. 2007;129:210-223.

52. Calder PC. $n-3$ polyunsaturated fatty acids, inflammation, and inflammatory diseases. Am J Clin Nutr. 2006;83:1505-1519.

53. Simopoulos AP. Omega-3 fatty acids in inflammation and autoimmune diseases. J Am Coll Nutr. 2002;21:495-505.

54. Sijben JWC, Calder PC. Differential immunomodulation with long-chain $n$-3 PUFA in health and chronic disease. Proc Nutr Soc. 2007;66:237-259

55. Larsson SC, Kumlin M, Ingelman-Sundberg M, et al. Dietary long-chain $n-3$ fatty acids for the prevention of cancer: a review of potential mechanisms. Am J Clin Nutr. 2004;79:935-945.

56. British Nutrition Foundation: $n-3$ Fatty Acids and Health [briefing paper]. London: British Nutrition Foundation; 1999.

57. Calder PC, Burdge GC. Fatty acids. In: Nicolaou A, Kokotos G, editors. Bioactive Lipids. Bridgewater: Oily Press; 2004:1-36.

58. Surette ME. The science behind omega-3 fatty acids. Can Med Assoc J. 2008; 178:177-180.

59. Calder PC, Yaqoob P. Omega-3 polyunsaturated fatty acids and human health outcomes. Biofactors. 2009;35:266-272.

60. Kremer JM, Lawrence DA, Petrillo GF, et al. Effects of high-dose fish oil on rheumatoid arthritis after stopping nonsteroidal antiinflammatory drugs. Clinical and immune correlates. Arthritis Rheum. 1995;38(8):1107-1114

61. Galarraga B, Ho M, Youssef HM, et al. Cod liver oil ( $n-3$ fatty acids) as an non-steroidal anti-inflammatory drug sparing agent in rheumatoid arthritis. Rheumatol (Oxford). 2008;47(5):665-669.

62. Dawczynski C, Hackermeier U, Viehweger M, et al. Incorporation of $n$-3 PUFA and $\gamma$-linolenic acid in blood lipids and red blood cell lipids together with their influence on disease activity in patients with chronic inflammatory arthritis - a randomized controlled human intervention trial. Lipids Health Dis. 2011;10:130.

63. Caughey GE, James MJ, Proudman SM, Cleland LG. Fish oil supplementation increases the cyclooxygenase inhibitory activity of paracetamol in rheumatoid arthritis patients. Complement Ther Med. 2010;18(3-4):171-174.

64. Sundrarjun T, Komindr S, Archararit N, et al. Effects of $n-3$ fatty acids on serum interleukin-6, tumour necrosis factor-alpha and soluble tumour necrosis factor receptor p55 in active rheumatoid arthritis. J Int Med Res. 2004;32(5):443-454.

65. Kolahi S, Ghorbanihaghjo A, Alizadeh S, et al. Fish oil supplementation decreases serum soluble receptor activator of nuclear factor-kappa B ligand/osteoprotegerin ratio in female patients with rheumatoid arthritis. Clin Biochem. 2010;43(6):576-580.

66. Das Gupta AB, Hossain AK, Islam MH, et al. Role of omega-3 fatty acid supplementation with indomethacin in suppression of disease activity in rheumatoid arthritis. Bangladesh Med Res Counc Bull. 2009;35(2): 63-68.

67. Dawczynski C, Schubert R, Hein G, et al. Long-term moderate intervention with $n$-3 long-chain PUFA-supplemented dairy products: effects on pathophysiological biomarkers in patients with rheumatoid arthritis. Br J Nutr. 2009;101(10):1517-1526.

68. Berbert AA, Kondo CR, Almendra CL, et al. Supplementation of fish oil and olive oil in patients with rheumatoid arthritis. Nutrition. 2005;21(2): 131-136.

69. Adam O, Beringer C, Kless T, et al. Anti-inflammatory effects of a low arachidonic acid diet and fish oil in patients with rheumatoid arthritis. Rheumatol Int. 2003;23(1):27-36.

70. Vitetta L, Ciccutinni F, Sali A. Alternative therapies for musculoskeletal conditions. Best Pract Res Clin Rheumatol. 2008;22(3):499-522.

71. Chong VH, Wang CL. Higher prevalence of gastrointestinal symptoms among patients with rheumatic disorders. Singapore Med J. 2008;49(5):419-424. 
72. Wolfe F, Hawley DJ. The comparative risk and predictors of adverse gastrointestinal events in rheumatoid arthritis and osteoarthritis: a prospective 13 year study of 2131 patients. J Rheumatol. 2000;27(7):1668-1673.

73. Coulson S, Vecchio P, Gramotnev H, Vitetta L. Green-lipped mussel (Perna canaliculus) extract efficacy in knee osteoarthritis and improvement in gastrointestinal dysfunction: a pilot study. Inflammopharmacology. 2012;20(2):71-76.

74. Wu GD, Chen J, Hoffmann C, et al. Linking long-term dietary patterns with gut microbial enterotypes. Science. 2011;334(6052):105-108.

75. Peltonen R, Kjeldsen-Kragh J, Haugen M, et al. Changes of faecal flora in rheumatoid arthritis during fasting and one-year vegetarian diet. $\mathrm{Br}$ J Rheumatol. 1994;33:638-643.

76. Peltonen R, Nenonen M, Helve T, et al. Faecal microbial flora and disease activity in rheumatoid arthritis during a vegan diet. Br J Rheumatol. 1997;36(1):64-68.

77. Vitetta L, Briskey D, Hayes E, et al. A review of the pharmacobiotic regulation of gastrointestinal inflammation by probiotics, commensal bacteria and prebiotics. Inflammopharmacology. Epub March 18, 2012.

78. Mackay IR, Rosen FS, Zinkernagel RM. Maternal antibodies, childhood infections, and autoimmune diseases. $N$ Engl J Med. 2001;345(18): 1331-1335.

79. Pineda M de L, Thompson SF, Summers K, et al. A randomized, double-blinded, placebo-controlled pilot study of probiotics in active rheumatoid arthritis. Med Sci Monit. 2011;17(6):CR347-CR354.
80. Mandel DR, Eichas K, Holmes J. Bacillus coagulans: a viable adjunct therapy for relieving symptoms of rheumatoid arthritis according to a randomized, controlled trial. BMC Complement Altern Med. 2010; $10: 1$.

81. Hatakka K, Martio J, Herranen M, et al. Effects of probiotc therapy on activity and activation of mild rheumatoid arthritis - a pilot study. Scand J Rheumatol. 2003;32:211-215.

82. Bengmark S, Martindale R. Prebiotics and synbiotics in clinical medicine. Nutr Clin Pract. 2005;20(2):244-261.

83. Calder PC, Albers R, Antoine JM, et al. Inflammatory disease processes and interactions with nutrition. Br J Nutr. 2009;101 Suppl 1:S1-S45.

84. Kotsirilos V, Vitetta L, Sali A, editors. A Guide to Evidence-Based Integrative and Complementary Medicine. Sydney: Elsevier; 2010.

85. Lindeberg S. Paleolithic diets as a model for prevention and treatment of Western disease. Am J Hum Biol. 2012;24(2):110-115.

86. Frei R, Lauener RP, Crameri R, O’Mahony L. Microbiota and dietary interactions - an update to the hygiene hypothesis? Allergy. 2012;67(4): 451-461.

87. Feng T, Elson CO. Adaptive immunity in the host-microbiota dialog. Mucosal Immunol. 2011;4:15-21.

88. Atarashi K, Tanoue T, Shima T, et al. Induction of colonic regulatory T cells by indigenous Clostridium species. Science. 2011;331:337-341.
Nutrition and Dietary Supplements

\section{Publish your work in this journal}

Nutrition and Dietary Supplements is an international, peer-reviewed, open access journal focusing on research into nutritional requirements in health and disease, impact on metabolism and the identification and optimal use of dietary strategies and supplements necessary for normal growth and development. The journal welcomes papers covering

\section{Dovepress}

original research, basic science, clinical \& epidemiological studies, reviews and evaluations, guidelines, expert opinion and commentary, case reports and extended reports. The manuscript management system is completely online and includes a very quick and fair peer-review system, which is all easy to use.

Submit your manuscript here: http://www.dovepress.com/nutrition-and-dietary-supplements-journal 